\title{
Spectrally-selective Time-resolved Emission through Fourier-filtering (STEF)
}

Anthony V. Sica, ${ }^{\mathrm{a}}$ Timothy L. Atallah, ${ }^{\mathrm{b}}$ and Justin R. Caram ${ }^{\mathrm{a}}{ }^{*}$

a) Department of Chemistry and Biochemistry, University of California, Los Angeles, 607 Charles E. Young Drive, Los Angeles, California 90095-1569, USA

b) Department of Chemistry and Biochemistry, Denison University, 500 West Loop, Granville, Ohio 43023, USA

Abstract: In this manuscript we demonstrate that directing the emitted photon stream from a fluorescent sample through a fixed path-length imbalanced Mach-Zehnder interferometer allows us to separate and resolve the dynamics of different emitters without the use of filtering optics. Our method, Spectrallyselective Time-resolved Emission through Fourier-filtering (STEF) takes advantage of a careful selection of interferometer position where one signal can be canceled (or enhanced) due to its unique spectral characteristics. STEF is straightforward to implement and provides a complementary approach to separate spectrally overlapped signals based on their coherence length and carrier frequency. We also discuss how one can implement STEF with an imperfect Mach-Zehnder interferometer, increasing the utility of this method, and demonstrate how Mach-Zehnder filtering can be used to image fluorophores in biologically relevant samples.

Introduction: Time-correlated single photon counting (TCSPC) uses emitted photon steam to measure (single) emitter lifetimes, ${ }^{1}$ intensity fluctuations ${ }^{2}$, classical/nonclassical photon correlations ${ }^{3}$, and material photophysical characteristics such as uncovering the relative polarization, ${ }^{4}$ phase,${ }^{5}$ frequency, ${ }^{6}$ and time of arrival. ${ }^{3,7,8}$ However, this often involves tradeoffs between spectral resolution, throughput, and experiment time which often limit our ability to resolve complex dynamics in heterogeneous mixtures. ${ }^{1,9-11}$ Previously we developed decay associated Fourier spectroscopy (DAFS) allowing us to both temporally and spectrally resolve photoluminescence by utilizing a scanning Mach-Zehnder (MZ) interferometer encoding simultaneous frequency and temporal information in the photon stream. ${ }^{11}$ By avoiding monochromator gratings and slits, DAFS is sensitive to weak signals, wavelength agnostic, can resolve octave spanning signals, while retaining the optical mode output of a microscope. However, DAFS is relatively slow, with a typical spectrum requiring 20-30 minutes to collect. To simply distinguish two signals at different frequencies, the use of specific optical filters is considerably more efficient.

Here we demonstrate that a MZ interferometer can act as a specifically tuned optical Fourier domain filter while at a fixed path length difference. By judiciously choosing the MZ path length difference we can maximally separate any two arbitrary (and overlapping) luminescence spectral signatures. We 
demonstrate the implementation of STEF (Spectrally-selective Time-resolved Emission through Fourierfiltering) for several different systems and show how it can be generalized and extended for applications in microscopy.

Photoluminescence in a Mach-Zehnder Interferometer In our implementation of MZ interferometry the incoming photon stream is directed through a beam splitter separating the light into two paths, where we introduce a variable delay (or phase shift) on one path using a stage and retroreflector (Figure 1A); for a more rigorous description see our previous method paper, and supporting information. ${ }^{11}$ By subtracting the

A
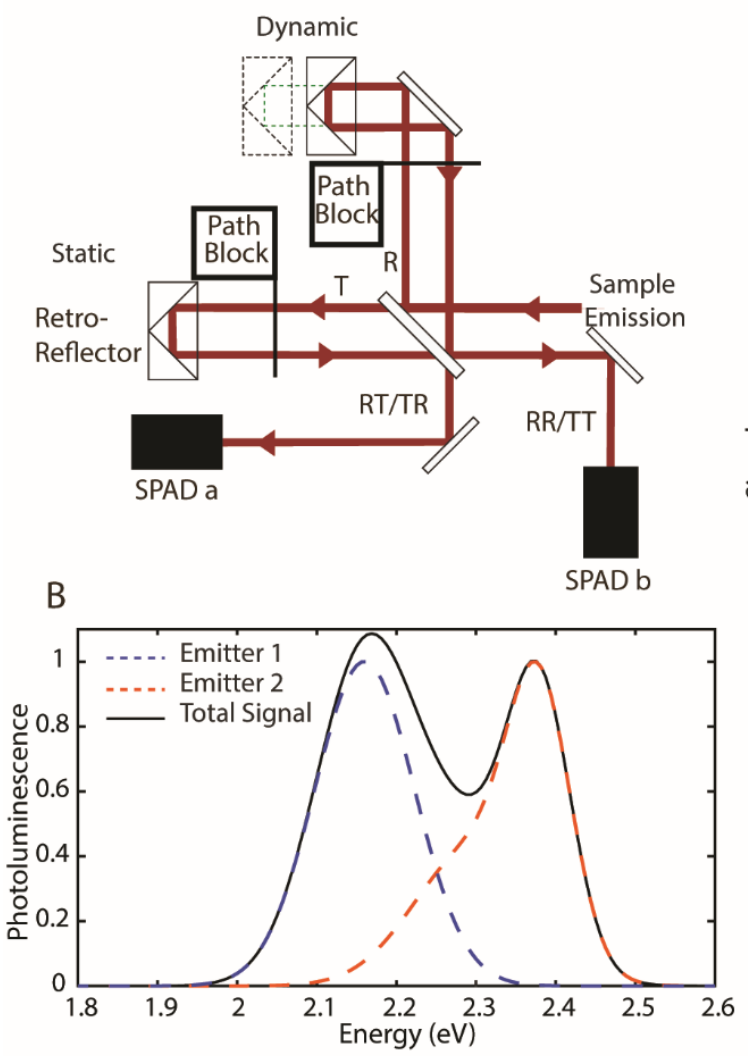

C

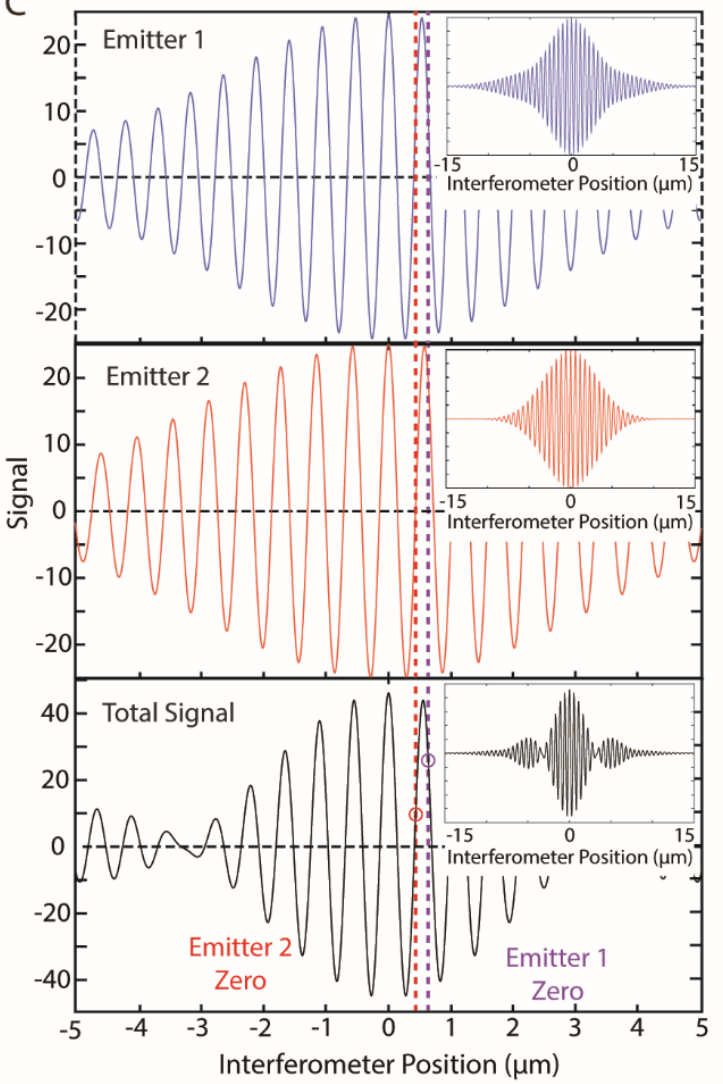

Figure 1 A. Folded Mach-Zehnder interferometer used for STEF. The path is blocked at specific points to retrieve combination of reflection (R) and transmission (T) terms to correct for any imbalances in the interference. The interferometer scans along a stage position on one arm labeled dynamic while the other remains static. B. Simulated example photoluminescence spectra of a solution with two emitters, the blue (emitter 1) and orange (emitter 2) dashed lines are the components from each emitter, with the black line as the sum of both components. C. The Fourier transform of each emitter (blue line: emitter 1; orange line: emitter 2) and sum (black line) demonstrating each emitter's zero crossing point (purple dashed line: emitter 1; red dashed line: emitter 2) in their corresponding $g_{1}(\tau)$. 
photon flux density, $I_{b}$, from one output of the interferometer from the other, $I_{a}$, we recover a signal proportional to the electric field first order correlation function, $g_{1}(\tau)$, which (for wide sense stationary signals) is the real part of the Fourier transform (FT) of the photon emission spectral intensity function, i.e. photoluminescence (PL) spectrum, $\sigma(\omega)$ illustrated for two emitters in Figure 1B. ${ }^{12,13}$

$$
I_{a}-I_{b} \propto \frac{\left\langle E^{*}(t) E(t+\tau)\right\rangle}{\left|\left\langle E^{*}(t) E(t)\right\rangle\right|}=g_{1}(\tau)=\operatorname{Re}\{F T[\sigma(\omega, \tau)]\}
$$

In Figure $1 \mathrm{C}$, we show the FT of the PL spectrum (e.g. the interferogram $g_{1}(\tau)$ ) for each emitter individually and the combined signal. In STEF the interferometer path length difference to a zero crossing (red and purple lines in Figure 1C) of one of the emitters filtering it out and leaving behind the signal from the other emitter. These zeroes may be determined from $g_{1}(\tau)$ of each individual chromophore. Thus, we obtain contrast between spectral components through a subtraction of detector signals, without the introduction of additional optical filter components. As all fluorescent signals have a carrier frequency, STEF can be applied to any lineshape and any arbitrary set of two (or more) emitter ensembles provided they have unique zero-crossings.

Correcting Imbalances: STEF relies on the cancellation of signals from each output of the MZ interferometer. However, the measured signal on each detector may arise due to differing efficiencies for each detector or imperfections reflection and transmission of the beamsplitter or other optical elements after the beamsplitter. We correct for these imbalances through by performing several simple calibration measurements. Briefly, the electric field at detector $a$ and $b$ are:

$$
\begin{aligned}
& E_{a}=R R E(t)+\operatorname{TTE}(t+\tau) \\
& E_{b}=R T E(t)+\operatorname{TRE}(t+\tau),
\end{aligned}
$$

where $\tau$ is the time separation induced by the path length difference. Here we assume that $\tau$ reflects a short time difference relative to the decay dynamics of the emitter (see supporting information section $\mathrm{S} 2$ ). $R$ and $T$ represent the transfer functions for the beamsplitter; $\mathrm{R}\left(\hat{R}|E(t)\rangle=\frac{\eta_{R} i}{\sqrt{2}}|E(t)\rangle\right)$ representing reflection and $\mathrm{T}\left(\widehat{T}|E(t)\rangle=\frac{\eta_{T}}{\sqrt{2}}|E(t)\rangle\right)$ representing transmission where $i$ accounts for the $\frac{\pi}{2}$ phase change introduced by 
the beamsplitter and the $\eta_{T}$ and $\eta_{R}$ are the efficiency terms of transmission and reflection being corrected. The observable intensity seen on each detector is:

$$
I_{a, b}=\frac{\eta_{a, b}}{2} \epsilon c\left|E_{a, b}(t)\right|^{2}
$$

where $\eta_{a, b}$ is the efficiency of the detector and the optics along each arm of the beamsplitter. Combining eq 5 and 6 with 7 we get what each detector measures:

$$
\begin{aligned}
& I_{a}=\frac{\eta_{a}}{2} \epsilon c|R|^{2}|T|^{2}\left(|E(t)|^{2}+|E(t+\tau)|^{2}+2 * \operatorname{Re}\left[E^{*}(t) E(t+\tau)\right]\right) \\
& I_{b}=\frac{\eta_{b}}{2} \epsilon c|R|^{2}|T|^{2}\left(|E(t)|^{2}+|E(t+\tau)|^{2}-2 * \operatorname{Re}\left[E^{*}(t) E(t+\tau)\right]\right)
\end{aligned}
$$

To correct for unknown reflection and transmission terms, we will block each part of the interferometer (see Figure 1A), and measure the intensity at $a$ and $b$. We will denote the intensity measure upon blocking each respective path as a series of $\mathrm{R}$ and $\mathrm{T}$ terms. For example, $I_{a T T}$ is the intensity measured at $a$ when the static retroreflector is blocked. Blocking paths 1 and 2 measures the following relationships:

$$
\begin{gathered}
I_{a R R}=\frac{\eta_{a} \eta_{R}^{2}}{2} \epsilon c\left(R R R^{*} R^{*}|E(t)|^{2}\right) \\
I_{a T T}=\frac{\eta_{a} \eta_{T}^{2}}{2} \epsilon c\left(T T T^{*} T^{*}|E(t+\tau)|^{2}\right) \\
I_{b R T}=\frac{\eta_{b} \eta_{R} \eta_{T}}{2} \epsilon c\left(R T R^{*} T^{*}|E(t)|^{2}\right) \\
I_{b T R}=\frac{\eta_{b} \eta_{T} \eta_{R}}{2} \epsilon c\left(T R T^{*} R^{*}|E(t+\tau)|^{2}\right)
\end{gathered}
$$

We can simplify equations 5 and 6 to show what each detector measures with an unblocked interferometer as follows:

$$
\begin{aligned}
& I_{a}=\left[I_{a R R}+I_{a T T}-\epsilon c \eta_{a}|R|^{2}|T|^{2} E^{*}(t+\tau) E(t)\right] \\
& I_{b}=\left[I_{b R T}+I_{b T R}+\epsilon c \eta_{b}|R|^{2}|T|^{2} E^{*}(t+\tau) E(t)\right]
\end{aligned}
$$


The total signal at each detector with the open interferometer is described as $I_{a / b}$ and the calibration terms (eq 7-10) are then subtracted from the total (eq. 11-12) to isolate our balanced signals: $\left.\pm \epsilon c \eta_{a / b}|R|^{2}|T|^{2} E^{*}(t+\tau) E(t)\right]$. In short, we collect signal with each path blocked as shown in Figure 1A. The total signal from each detector is subtracted from the respective detector at each blocked path to calibrate any imbalance. Finally, the calibrated signal from each detector is then subtracted to give us the isolated signal. It should be noted that within our subtraction the detector efficiencies, $\eta_{a}$ and $\eta_{b}$ remain. Nonetheless, the sum of equations 7 and 8 can be normalized and matched the sum of equations 9 and 10 to find the detector efficiencies.
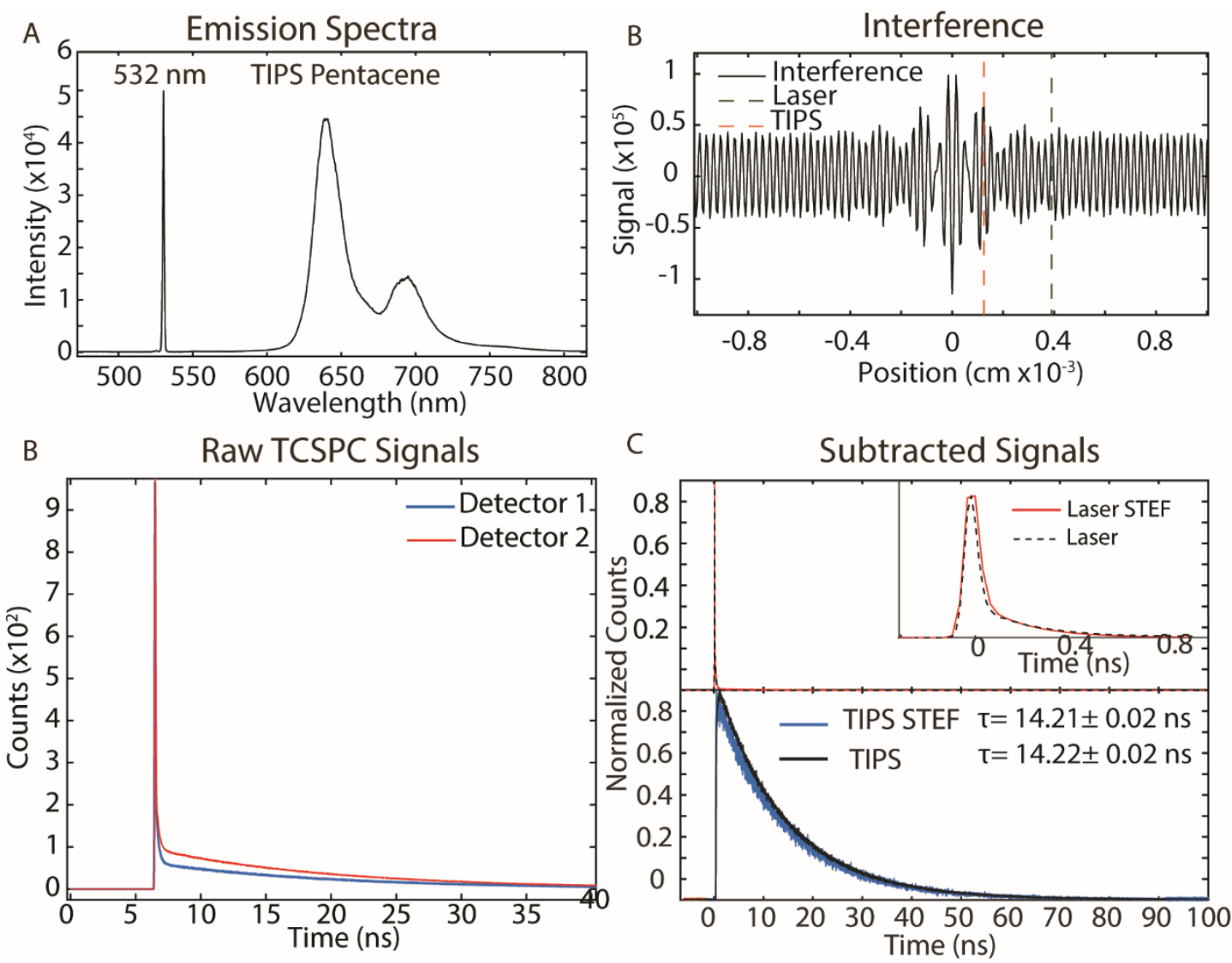

Figure $2 \mathrm{~A}$. The emission spectra of the laser and emitter, B. the respective interference collected with the MZ interferometer. C. The TCSPC lifetime on each detector retrieved before correction. D. After subtraction and correction the signals can successfully separate yielding the laser limited instrument response function and the lifetime of TIPS pentacene. Data is fit to a single exponential.

Separating Excitation from Emission: As a proof of principle and example of applicable use, we 
implement STEF to separate laser scatter from sample PL (Figure 2). Using a picosecond pulsed diode laser $532 \mathrm{~nm}$ we excite TIPS-pentacene (6,13-Bis(triisopropylsilylethynyl)pentacene) and measure TCSPC traces through the MZ interferometer. In Figure 2A we show the spectra of the laser and emitter, TIPSpentacene) used and the respective interference collected with the $\mathrm{MZ}$ interferometer. The interferometer is set to interference zero positions for laser and fluorescence signal respectively, and we record TCSPC traces with the interferometer open (Figure 2B), the static retroreflector blocked, and the moving retroreflector blocked (calibration data shown in Figure S3). After subtraction and correction, the laser and TIPS-pentacene signals are successfully isolated (Figure 2C). The laser signal as expected matches instrument response function. The STEF resolved TIPS-pentacene signal is identical to the unmixed TIPSpentacene lifetime decay giving the same lifetimes within instrumental error. This demonstrates the powerful utility of STEF in filtering out laser scatter, which often can be detrimental in imaging and spectroscopic measurements.

Separation of Two Emitters: We next employ STEF with a more complex system: a hexanes solution of rubrene and TIPS-pentacene (structures and spectra in Figure 3A). This demonstrates the generality of

A

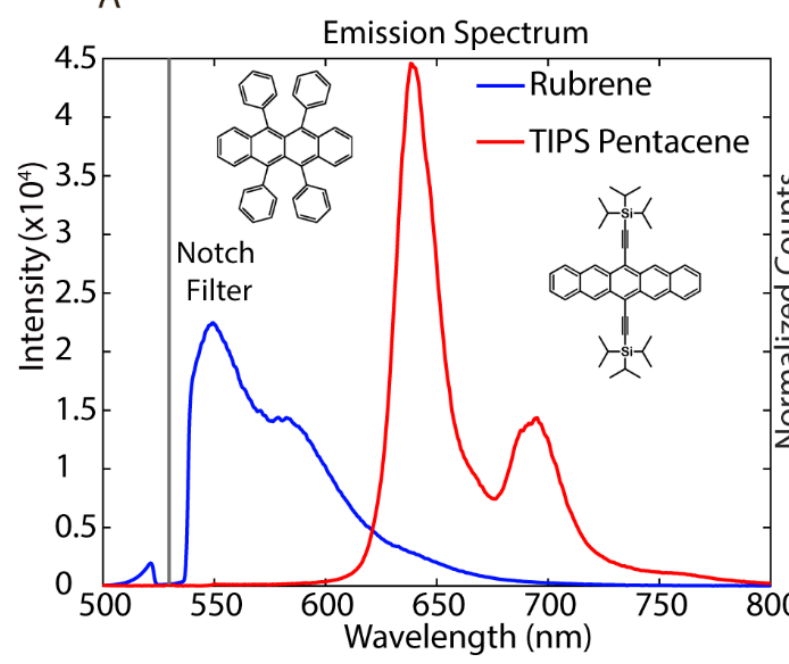

B

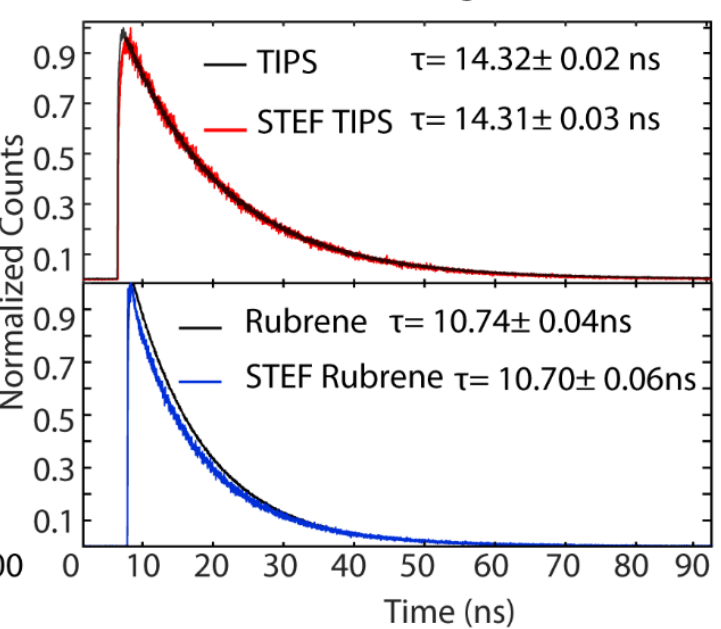

Figure 3 A. Emission spectrum of the two-emitter system, Rubrene and TIPS -pentacene. A 533-notch filter was used throughout the experiment to filter out the laser and maintain a two-component system. The two emitters were excited using a 532-diode laser. B. The resulting lifetimes from the subtraction each compared with the lifetimes of the emitters unmixed. The similar lifetimes demonstrate STEF's ability to successfully subtract and isolate emissions even with molecules of similar lifetimes 
STEF's ability to separate signals of arbitrary spectral shape, even with significant spectral overlap. We observe the dynamics of each component separately without the use of any additional filters except a 533 notch filter to remove any laser scattering. The STEF measured lifetime traces of each component are identical to their unmixed samples (Figure 3B). The exponential fits of both the STEF and sole chromophore solutions lifetimes again agree within experimental error, successfully showing the generality of STEF for mixed chromophore systems.

Separation of Fluorescent Channels in Cellular Imaging: Finally, we show the breadth of STEF's utility by implementing the method in the context of imaging. Using a homebuilt inverted microscope setup

A

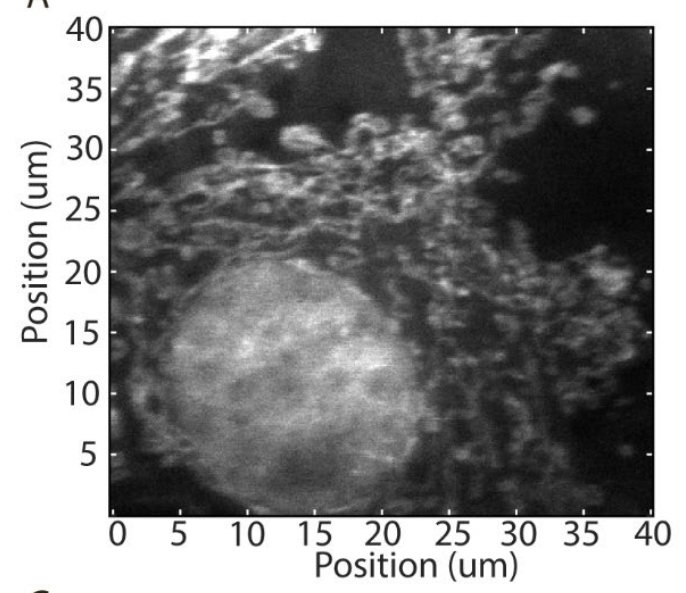

C

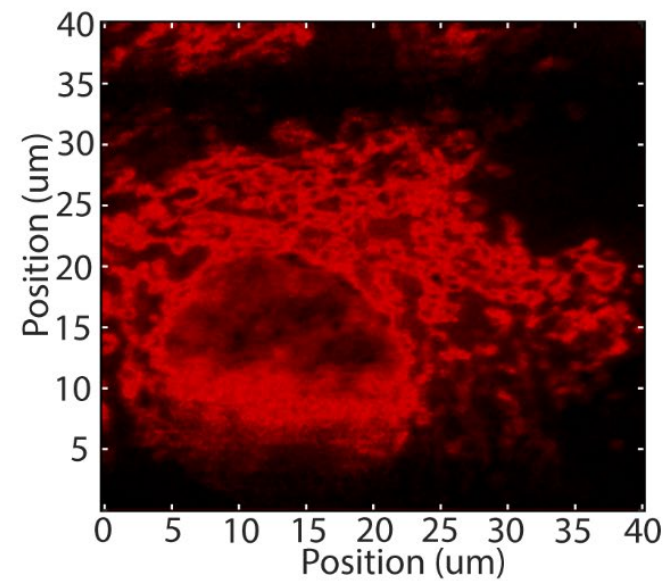

B
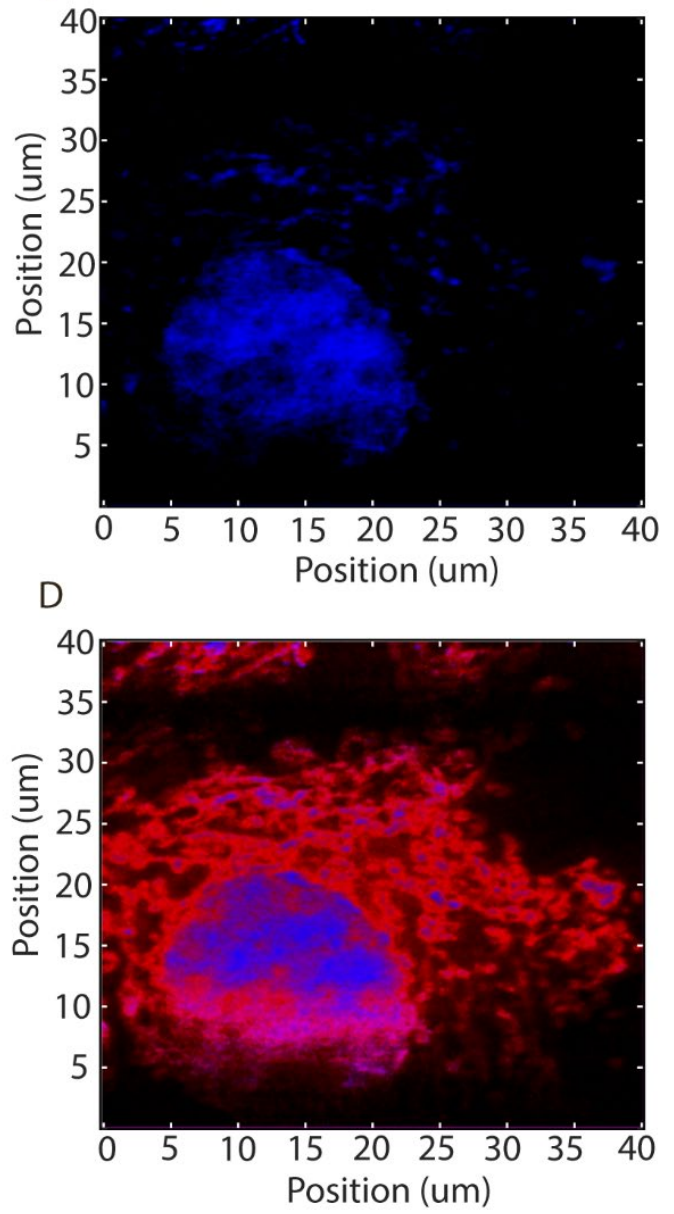

Fig. 4 A. Imaging of BPAE Cells using scanning confocal microscopy prior to STEF. Only MitoTracker ${ }^{\mathrm{TM}}$ Red and DAPI are being excited by the $532 \mathrm{~nm}$ laser. B. Scanning image when detectors are subtracted to filter and remove MitoTracker ${ }^{\mathrm{TM}}$ Red CMXRos channel leaving the nucleus with DNA stained with DAPI C. Scanning image at a different stage position where detector subtraction filters and remove DAPI leaving the mitochondria in the cytosol stained with MitoTracker ${ }^{\mathrm{TM}}$ Red CMXRos. D. Combined image overlay of B and C. 
(details in the supporting information), and a notch filter to block direct laser scatter, we performed confocal microscopy to simultaneously image multiple fluorescent channels of stained bovine pulmonary artery endothelial cells. The total image is shown in (Figure 4A). Following the same procedure described previously, we separate the fluorescence from the 4',6-diamidino-2-phenylindole, DAPI stain (DNA signal, shown in Fig 4B) from the MitoTracker ${ }^{\mathrm{TM}}$ Red CMXRos stain (mitochondria signal Figure 4C) without filters. Figure 4D shows STEF correctly resolves the blue DNA to the cell nucleus from the surrounding red mitochondria and would allow for the tuning of whatever signal ensembles appropriate for the system, confirming its general scope in biological or other imagine.

Conclusion: As demonstrated, STEF can be a powerful tool to separate spectral signatures by leveraging differences in the carrier frequency and coherence length. This avoids difficulties often seen in systems with emission overlap and complicated line shapes which lead to signal contamination. The practical consideration of STEF's contrast is set by the difference in carrier frequency (or the accumulated phase difference between signals), and their coherence length (the width of the spectrum). Therefore, STEF works best when two signals are separated in energy (carrier frequency), and are narrow (large coherence lengths), paralleling traditional filters. With these factors in mind, STEF presents the advantage of overall tunability and specificity to arbitrary desired signals that may even have overlapping spectral signatures without requiring specialized filters. Hence, we were able to demonstrate separation of the emission from a laser and chromophore, two separate chromophores and imaging of two channels in a stained cellular environment. These show STEF's wide utility across disciplines in spectroscopy, solution analysis, material science. and biological imaging. 
Data Availability Statement: The data that support the findings of this study are available from the corresponding author upon reasonable request.

Funding and Acknowledgements: AVS, TLA, JRC would like to acknowledge funding from the National Science Foundation, Career Grant CHE-1945572 and the ACS Petroleum Research Fund, 62717-DNI6

\section{References}

1. Jian Cui, P. Beyler, A., S. Bischof, T., B. Wilson, M. W. \& G. Bawendi, M. Deconstructing the photon stream from single nanocrystals: from binning to correlation. Chem. Soc. Rev. 43, 12871310 (2014).

2. Schlegel, G., Bohnenberger, J., Potapova, I. \& Mews, A. Fluorescence Decay Time of Single Semiconductor Nanocrystals. Phys. Rev. Lett. 88, 137401 (2002).

3. Rigler, R. \& Elson, E. S. Fluorescence Correlation Spectroscopy: Theory and Applications Google Books. Springer (2012). Available at:

https://books.google.com/books?id=iAH8CAAAQBAJ\&printsec=frontcover\&source=gbs_ge_su mmary_r\&cad $=0 \# \mathrm{v}=$ onepage $\& \mathrm{q} \& \mathrm{f}=$ false. $($ Accessed: 8 th July 2021)

4. Kliger, D. S., Lewis, J. W. \& Randall, C. E. Polarized Light in Optics and Spectroscopy - David S. Kliger, James W. Lewis - Google Books. Academic Press, Inc (1990). Available at: https://books.google.com/books?hl=en\&lr=\&id=ZPzC9GHdsJcC\&oi=fnd\&pg=PP1\&dq=polarize $\mathrm{d}+$ spectroscopy\&ots=WcRAAfKZJ0\&sig=GkQFGDgQcz75dJSU4vAWupUayic\#v=onepage\&q= polarized spectroscopy\&f=false. (Accessed: 8th July 2021)

5. Terasaki, A., Tokunaga, E. \& Kobayashi, T. Frequency-domain interferometer for femtosecond time-resolved phase spectroscopy. Opt. Lett. Vol. 17, Issue 16, pp. 1131-1133 17, 1131-1133 (1992).

6. Valeur, B. \& Berberan-Santos, M. N. Molecular Fluorescence : principles and applications. 569 
(2013).

7. Schlawin, F. Entangled photon spectroscopy. J. Phys. B At. Mol. Opt. Phys. 50, 203001 (2017).

8. Loumaigne, M., Vasanthakumar, P., Richard, A. \& Débarre, A. Time-of-Flight Photon Spectroscopy: A Simple Scheme To Monitor Simultaneously Spectral and Temporal Fluctuations of Emission on Single Nanoparticles. ACS Nano 6, 10512-10523 (2012).

9. Crouch, C. H. et al. Facts and Artifacts in the Blinking Statistics of Semiconductor Nanocrystals. 10, 1692-1698 (2010).

10. Ambrose, W. P. \& Moerner, W. E. Fluorescence spectroscopy and spectral diffusion of single impurity molecules in a crystal. Nat. 19913496306 349, 225-227 (1991).

11. Atallah, T. L. et al. Decay-Associated Fourier Spectroscopy: Visible to Shortwave Infrared TimeResolved Photoluminescence Spectra. J. Phys. Chem. A 123, 6792-6798 (2019).

12. Loudon, R. Classical theory of optical fluctuations and coherence. in The Quantum Theory of Light 82-124 (Oxford University Press: New York, 2000).

13. Griffiths, P. R. \& De Haseth, J. A. Fourier Transform Infrared Spectrometry: Second Edition. Fourier Transform Infrared Spectrom. Second Ed. 1-529 (2006). doi:10.1002/047010631X 